\title{
Modeling of active yaw systems for small and medium wind turbines
}

\author{
Simon De Zutter, Jeroen D. M. De Kooning, Arash E. Samani, Jens Baetens and Lieven Vandevelde \\ Ghent University (Belgium), Electrical Energy Laboratory (EELAB) \\ Department of Electrical Energy, Metals, Mechanical Constructions \& Systems (EEMMeCS) \\ Contact: Jeroen.DeKooning@UGent.be
}

\begin{abstract}
This paper proposes the use of an active yaw system to protect small and medium wind turbines in the high wind zone from overloading. The active yaw system turns the rotor partially out of the wind to decrease the turbine power. The dependency of the power on the yaw angle is modeled by the third power of the cosine of the yaw angle. A proper controller is configured based on on-off control of the yaw mechanism. A supplementary rotor speed control is required to avoid static instability. The proposed power limitation strategy is simulated in dynamic situations. The step response simulations show that a yaw rate of the order of $3 \%$ is required to properly limit the power. Simulations using a realistic wind profile demonstrate that the system can cope with the fluctuating nature of the wind.
\end{abstract}

Keywords-Wind energy, small and medium wind turbines, power limitation, active yaw control

Primary conference subject area-Renewable energy systems

\section{INTRODUCTION}

One of the major challenges of the twenty-first century is global warming caused by excessive carbon emissions. In response to the concerns about the climate, renewable energy technologies are researched and installed extensively in the last two decades. One of the biggest renewable energy sources is wind energy. The gross of the wind turbine capacity installed worldwide consists of MW class wind turbines. The technology of large wind turbines has reached a mature phase. To the contrary, small and medium wind turbines (SMWTs) do not have reached this point yet. Therefore, in this work, efforts are made to develop better systems for this class of wind turbines.

When wind turbines operate at high wind speeds, a high load is imposed on the generator and converter. If the wind speed exceeds the rated value, the power must be limited to prevent damage. For larger turbines, well performing power limiting mechanisms (PLMs), such as pitch control and active stall control, are already developed and installed. For small and medium wind turbines these systems are less common and the power control usually relies on passive stall control. The problem of passive stall control is that it is not able to limit the power accurately. Fig. 1 presents the power as a function of wind speed in a passive stall controlled wind turbine [1]. There is an overshoot of the power with respect to the rated value at wind speeds slightly above the rated wind speed. For even higher wind speeds, the power decreases again and eventually becomes lower than the rated power. Therefore power control in the high wind zone is not optimal. When a turbine is expected to operate frequently in the high wind zone, more effective PLMs are desired. This paper explores the possibilities of an active yaw system combined with rotor speed control for power control in the high wind zone for SMWTs. The normal function of the active yaw system is to track the direction of the wind. Also in extreme conditions such as a storm, the rotor is yawed $90^{\circ}$ out of the wind. However, in this paper the active yaw system is used to rotate the rotor partially out of the wind to maintain the power at rated conditions in the high wind zone.

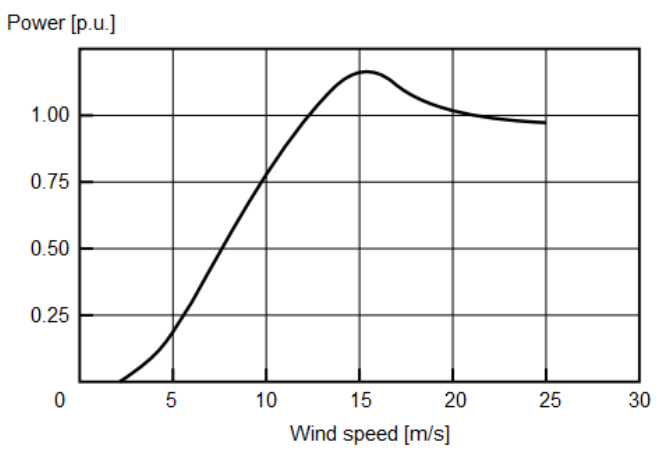

Fig. 1. Power curve using passive stall [1]

\section{WIND TURBINE MODEL}

The wind turbine system considered in this paper is of the three-bladed horizontal axis type. Fig. 2 gives an overview of the most common topology based on a Permanent Magnet Synchronous Generator (PMSG). The turbine (a) drives the PMSG (b). Since the shaft speed is variable, the PMSG produces an ac with a variable frequency and amplitude, i.e., 'wild ac'. The wild ac is converted to a dc voltage by the active rectifier (c) while performing the MPPT. An inverter (d) then injects an ac current with a constant frequency into the grid (e).

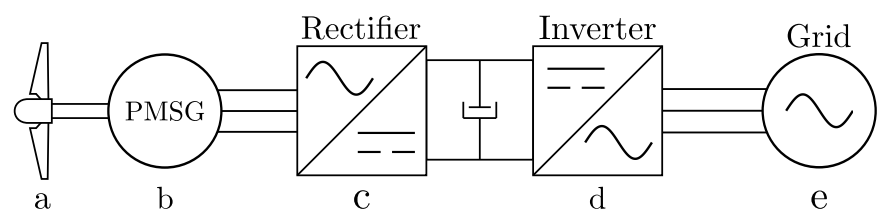

Fig. 2. Overview of the considered wind turbine system

\section{A. General model}

The mechanical power of the turbine $P_{t}$ is given by: 


$$
P_{t}=\frac{1}{2} \rho \pi R^{2} v^{3} C_{p}
$$

where $\rho$ is the air density, $R$ is the turbine radius and $v$ is the wind speed. The factor $C_{p}$ is the power coefficient of the turbine, which is determined by the Tip-Speed Ratio (TSR) $\lambda$. The pitch system is not considered here. The TSR characterizes the air flow around the blades and is defined by:

$$
\lambda=\frac{R \Omega}{v}
$$

where $\Omega$ is the rotational speed of the turbine. The relation between the power coefficient $C_{p}$ and the TSR $\lambda$ is determined by the shape of the blade. Here, an empiric $C_{p}$ relation from [4], [5] is used to model the blades:

$$
C_{p}=\left(\frac{110}{\lambda}-9.96\right) e^{\left(\frac{-18.4}{\lambda}+0.055\right)}
$$

This power coefficient $C_{p}$ is shown graphically in Fig. 3 . The power coefficient reaches a maximum value of 0.44 for a TSR of 6.91, which is the maximum power point (MPP). The MPP is the most favorable operating point of the turbine as the power output is maximized here.

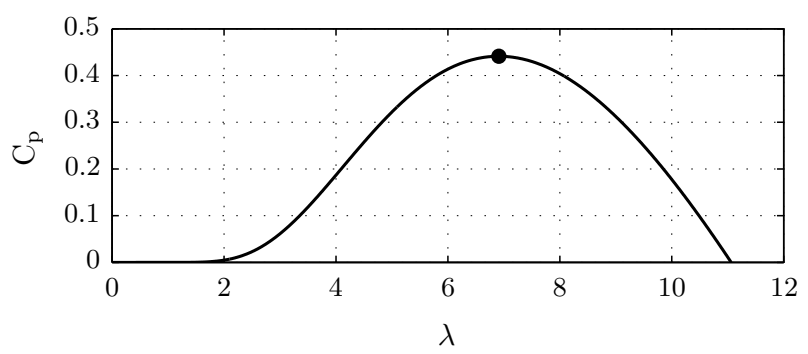

Fig. 3. Empiric $C_{p}$ curve [4], [5]

\section{B. Modeling of the active yaw system}

In literature, different expressions are used for the turbine power in case the yaw angle $\gamma$ is different from zero. Fig. 4 illustrates the definition of the yaw angle $\gamma$ [11]. It is the angle between the direction of the oncoming wind speed and the rotor axis.

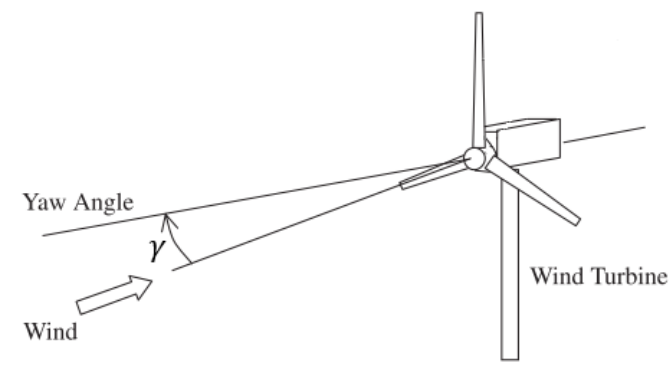

Fig. 4. Definition of the yaw angle [11] angle:

$$
P_{t}=\frac{1}{2} \rho \pi R^{2} v^{3} C_{p} \cos \gamma
$$

This equation is based on the fact that the mass flux of the wind through the rotor surface is decreased by $\cos \gamma$ when the normal of the rotor surface has an angle $\gamma$ with the wind direction. Indeed, the power extracted from the wind is proportional to the mass flux of the wind and consequently the power decreases also by $\cos \gamma$. However, this reasoning does not take into account the fact that the efficiency of the blades decreases under yawed inflow conditions. When there is a nonzero yaw angle $\gamma$, the wind does not strike the leading edge of the blade orthogonally. Hence, the blade does not generate the same lift forces as it would generate with orthogonal inflow, i.e., the lift forces decrease. Therefore, it is argued that for the calculation of the power, only the orthogonal component of the wind should be used. Therefore the turbine power becomes [9], [10]:

$$
P_{t}=\frac{1}{2} \rho \pi R^{2} v^{3} C_{p} \cos ^{3} \gamma
$$

Wind tunnel tests were performed on a turbine rotor under yawed inflow conditions [11]. Pressure distributions over the blade section were measured in order to compute forces and power. It was observed that the measurements are close to the curve of $\cos ^{3} \gamma$, i.e., equation (5). However, in [11] it was observed that this model is less accurate when tip speed ratios become low.

In reality, the situation is complex. To obtain the real power that is produced by the rotor accurately, a detailed study using computational fluid dynamics (CFD) should be performed. It is beyond the scope of this work to perform such calculations. For the discussion to follow, (5) will be adopted to represent the turbine power under yawed conditions. However, care should be taken when simulating low tip speed ratios.

\section{Yaw control for power limitation}

This paper focusses on the operation of a small wind turbine for wind speeds above the nominal value. In this operating range, the goal is to keep the power limited to the rated value, so that the drivetrain components are not damaged by excessive speed, torque, voltage or current. By combining (1), (3) and (5), it can be analytically derived which yawing angle $\gamma$ is required in function of the wind speed, to keep the power equal to the rated value:

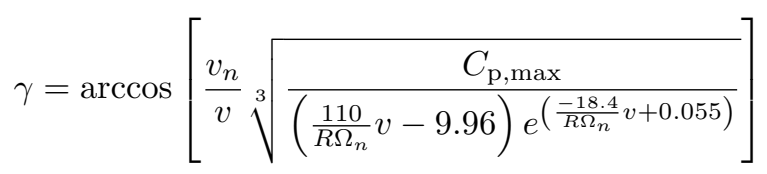

Fig. 5 shows this curve for a rated wind speed $v_{n}$ of $11 \mathrm{~m} / \mathrm{s}$, a blade length of $1.54 \mathrm{~m}$ and a maximum power coefficient $C_{\mathrm{p}, \max }$ of 0.44 . The yaw angle rises steeply when the wind speed exceeds the nominal value. It reaches a maximum and then decreases again. The reason for the decrease of the yaw angle is the increasing stall of the blades with increasing wind speed. Hence, less yaw misalignment is required to limit the 
power. The range of the yaw angle is between $0^{\circ}$ and $40^{\circ}$. This means, if the wind speed would rapidly increase to the value of $19 \mathrm{~m} / \mathrm{s}$ in this case, the yaw system would be required to rotate the nacelle $40^{\circ}$ out of the wind. As the rotation speed of the yaw system is limited, it will take some time to attain the proper yaw angle. The maximum rotational speed of the nacelle that the yaw system can deliver will be a decisive factor in the power limiting ability of the active yaw control strategy.

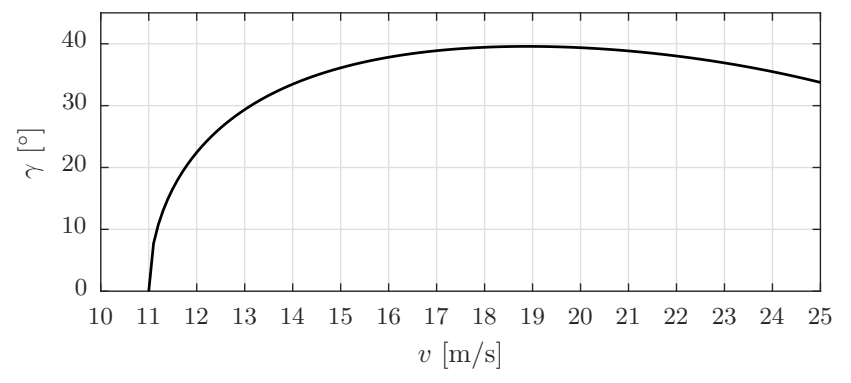

Fig. 5. Evolution of the yaw angle

\section{YAW CONTROL SYSTEM}

Implementing the active yaw control strategy to limit the power requires a proper controller. The yaw mechanism consists of an electrical motor that drives a worm gear. Including the details of the mechanism implies that the motor dynamics and control should be considered. Moreover, during the yaw motion, counteracting torques emerge from the wind that impacts the rotor and the nacelle. These torques are not straightforward to calculate. In order to focus on the inherent properties of the yaw strategy, abstraction is made of the yaw mechanism. The yaw mechanism is simplified to a system that imposes a certain yaw rate $\dot{\gamma}$. The yaw rate can be set instantaneously and no start up of the motor or counteracting torques are considered. The rate is however limited as the yaw mechanism has a limited amount of power. In that way, the analysis is not burdened by the details of the mechanism and focus can go to comparing the active yaw strategy with other power limiting strategies based on their fundamental properties rather than their practical ones.

\section{A. Control of the yaw angle}

The goal of the controller is to obtain an appropriate yaw angle $\gamma$ for wind speeds above the rated value such that power is limited. In order to do so, the yaw angle is increased when the power exceeds the nominal value. When the power decreases again, the yaw angle is decreased accordingly. A measurement of the wind speed is not necessary. The measurement of the power provides sufficient information for the controller to make proper decisions. In principle, the turbine power $P_{t}$ could be used as input for the controller. However, it is more convenient to use the electrical power $P_{e}$ as this requires the measurement of electrical quantities instead of mechanical ones. Sensors for measuring current and voltage are easier to construct and are consequently less costly than sensors for speed and torque. Moreover, they are often already present in the converter.

The electrical power $P_{e}$ is compared to the nominal value $P_{e, n}$. In case of deviation, control action is required. A PI controller could be an appropriate solution to generate the reference values for the yaw angle. However, using PI control in combination with rate limited actuators causes problems. More specifically, the integral term is troublesome. When a yaw angle is set, the nacelle is rotated to this angle at a constant rate. Because during the yaw motion the electrical power $P_{e}$ still differs from the nominal value $P_{e, n}$, the error is continued to be integrated. This causes the integral action to grow too much and unstable oscillations result. It is in fact similar to integral windup when the system is confronted with physical limits. However, this time, there is no constant limit such that the anti-windup schemes cannot be applied here.

Since PI control is not suited, an other control method must be applied. The control method applied is the on-off control with a dead zone. When the electrical power $P_{e}$ is in the interval $\left[P_{e}-\delta, P_{e}+\delta\right]$ no control action is performed. This interval is called the dead zone. When the electrical power $P_{e}$ exceeds the value $P_{e, n}+\delta$, the yaw mechanism is commanded to rotate at its maximum speed $\dot{\gamma}_{\text {max }}$, increasing the yaw angle $\gamma$. Similarly, when the electrical power $P_{e}$ is below $P_{e, n}-\delta$, the yaw mechanism is commanded to decrease the yaw angle $\gamma$ at maximum speed $-\dot{\gamma}_{\max }$. In this way, power is regulated until it reaches the dead zone. The width of the dead zone is determined by the parameter $\delta$. If this parameter is chosen small, the electrical power $P_{e}$ will approach closely the nominal value $P_{e, n}$. However, it will also result in excessive oscillations of the yaw angle. A conscious choice for $\delta$ should be made in order to control close enough to the nominal power $P_{e, n}$ but without inducing too much oscillations of the yaw system.

\section{B. Control of the rotor speed}

In principle, to limit the power and speed to the nominal values, no control of the rotor speed $\Omega$ is required. In the high wind zone, the generator applies the nominal torque. The yaw controller sets the appropriate yaw angle $\gamma$ and the rotor speed $\Omega$ will automatically converge to the nominal value. However, an examination of the torque-speed characteristic of the wind turbine renders that the operating point in the high wind zone is statically unstable. The torque-speed characteristic is plotted in Fig. 6 for $v=v_{n}$ and $v=1.5 v_{n}$. For $v=1.5 v_{n}$, i.e., in the high wind zone, the yaw system is active and the yaw angle $\gamma$ is set according to (6). The operating point can be found at the one per unit values of the turbine torque $T_{t}$ and the rotor speed $\Omega$. It is observed that the operating point at the curve of $v=1.5 v_{n}$ is indeed statically unstable. When there is a disturbance to the rotor speed $\Omega$ and it increases for example a small amount, then the turbine torque $T_{t}$ increases. This implies again an increase of the rotor speed $\Omega$ if the generator torque $T_{g}$ stays constant. Consequently, the operating point diverges from the desired operating point. Similar reasoning holds for a small decrease of the rotor speed $\Omega$.

To avoid the static instability, a control of the rotor speed $\Omega$ is required. This control can be achieved by a PI controller. In the high wind zone, the rotor speed $\Omega$ is controlled to the nominal value. The PI controller generates a current reference $I$ for the converter based on the deviation of the rotor speed $\Omega$ from the nominal value. The current setting $I$ is translated into a generator torque $T_{g}$. Hence, the speed can be controlled by appropriate set values for the current $I$. 


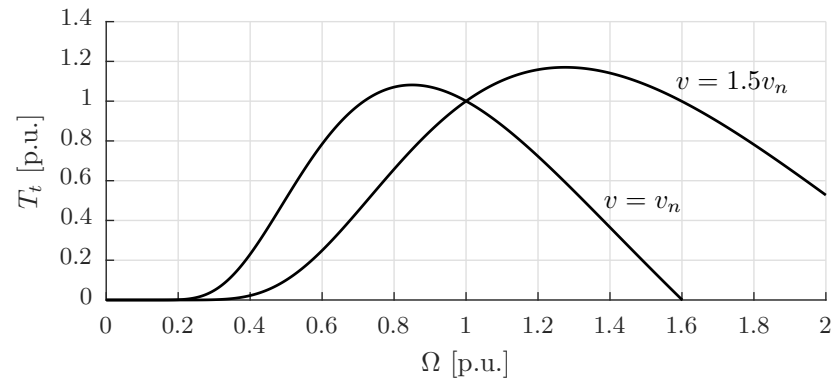

Fig. 6. Torque-speed characteristics for $v=v_{n}$ and $v=1.5 v_{n}$. In the high wind zone, the operating point is statically unstable

\section{Simulation RESUlts}

Simulations are performed of the wind turbine system equipped with a yaw mechanism. The strategy of limiting the power in the high wind zone using the yaw mechanism is tested in a dynamic situation. From these simulations, the performance of the active yaw strategy can be evaluated. The relevant parameters of the wind turbine system are given in Table I. The width of the dead zone $\delta$ is presented relatively to the nominal electrical power $P_{e, n}$.

TABLE I. SYSTEM PARAMETERS

\begin{tabular}{cccccc}
\hline$P_{e, n}$ & $v_{n}$ & $\Omega_{n}$ & $R$ & $\rho$ & $\delta / P_{e, n}$ \\
\hline$[\mathrm{kW}]$ & {$[\mathrm{m} / \mathrm{s}]$} & {$[\mathrm{rpm}]$} & {$[\mathrm{m}]$} & {$\left[\mathrm{kg} / \mathrm{m}^{3}\right]$} & {$[\%]$} \\
\hline 2.1 & 11 & 471 & 1.54 & 1.225 & 2.5 \\
\hline
\end{tabular}

\section{A. Step response}

The step response is obtained from applying a step in the wind profile. It is not a realistic situation that the wind changes in steps. Nevertheless it is worthwhile to study the step response as this allows to study the dynamic behavior of the wind turbine quantitatively. The $2 \%$ settling time $t_{s}$ of the rotor speed $\Omega$ is used for the quantification of the dynamic behavior. The settling time $t_{s}$ of the rotor speed $\Omega$ is defined as the time needed for $\Omega$ to reach and stay in a certain error region around the steady state value $\Omega_{s s}$ :

$$
\forall t>t_{s}:(1-c) \Omega_{s s}<\Omega(t)<(1+c) \Omega_{s s}
$$

In case of the $2 \%$ settling time, the quantity $c$ equals 0.02 .

The results of a step response simulation are presented in Fig. 8. The wind speed $v$ jumps from the nominal value $v_{n}=$ $11 \mathrm{~m} / \mathrm{s}$ to $v=14 \mathrm{~m} / \mathrm{s}$ at time $t=3 \mathrm{~s}$. The yaw rate $\dot{\gamma}$ is set at $5 \%$ s. The generator torque is limited because of the limited current. Consequently, the rotor speed rises quickly. Meanwhile the yaw mechanism increases the yaw angle in order to limit the power. Because the yaw rate $\dot{\gamma}$ is limited, it takes some time before the yaw angle reaches the desired magnitude. Before the yaw angle settles at its final value, there is a small overshoot. The settling time $t_{s}$ is $8.1 \mathrm{~s}$.

It is interesting to study the settling time $t_{s}$ as a function of the yaw rate $\dot{\gamma}$. The settling time $t_{s}$ will decrease if $\dot{\gamma}$ increases. However, a high yaw rate $\dot{\gamma}$ implies a yaw mechanism of a higher power rating. Consequently, the costs of the yaw mechanism will rise. Hence, one will choose a yaw rate $\dot{\gamma}$ that is as low as possible but still assures proper power limitation, i.e., has an acceptable settling time.

The settling time $t_{s}$ is calculated for step responses of different step sizes $\Delta v$. The result is presented in Fig. 7. For yaw rates $\dot{\gamma}$ smaller than $1 \%$ s the settling time $t_{s}$ becomes excessively large. When the yaw rate increases, the settling time decreases steeply. Increasing the yaw rate even more, the settling time still decreases but the effect of the increased yaw rate is less. In general, it can be inferred from Fig. 7 that the yaw rate $\dot{\gamma}$ must be of the order of $3 \%$. If the yaw rate $\dot{\gamma}$ is chosen lower, the settling time becomes large rapidly. Choosing a yaw rate $\dot{\gamma}$ substantially higher than $3 \%$ is not recommended as the price of the yaw mechanism will go up and the added value for the dynamic performance is limited.

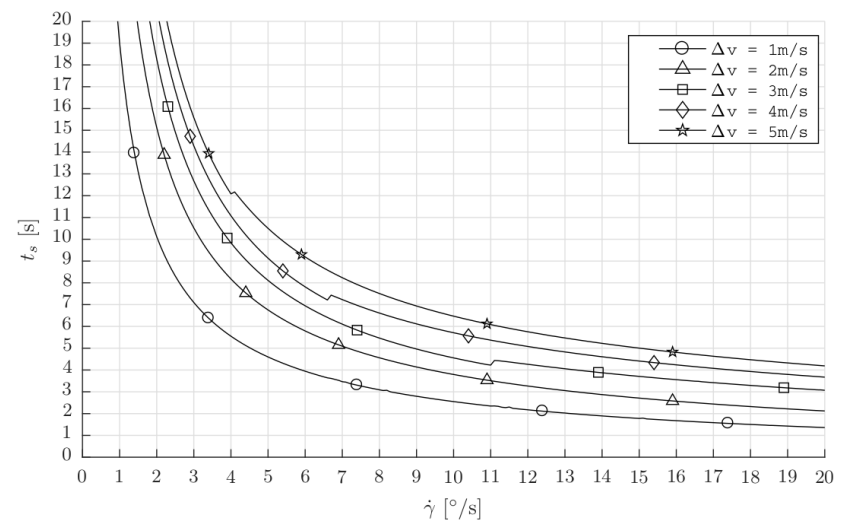

Fig. 7. Settling time in function of yaw rate

\section{B. Realistic Simulation}

The step response simulations are convenient for a quantitative study of the active yaw strategy. However, a wind profile existing out of steps is not a realistic situation. In reality, the wind will exhibit a highly fluctuating wind speed. Therefore, simulations are performed using a realistic wind profile. From these simulations it can be observed if the active yaw strategy can cope with fluctuating wind speeds. The simulation results are presented in Fig. 9. A yaw rate $\dot{\gamma}=2.5^{\circ} \mathrm{s}$ is applied. This corresponds with a settling time $t_{s}$ of $15 \mathrm{~s}$ at a step size $\Delta v=3 \mathrm{~m} / \mathrm{s}$ as can be seen from Fig. 7 .

In order to handle the rapid increasing wind speed at the beginning of the simulation, the yaw angle is increased by the controller. During this transient period, the electrical power $P_{e}$ and the rotor speed $\Omega$ exceed the nominal values. The maximal occurring values of electrical power $P_{e}$ and speed $\Omega$ are near $3200 \mathrm{~W}$ and $65 \mathrm{rpm}$ respectively. These values correspond to $150 \%$ and $132 \%$ of the nominal values respectively. The transient has a duration of approximately $12 \mathrm{~s}$. If the drivetrain is not able to cope with such transients, the yaw rate $\dot{\gamma}$ must be chosen higher. After the transient, the active yaw strategy succeeds in maintaining the power to the nominal value. The fluctuation of the power in the high wind zone is low. Also the fluctuation of the yaw angle is limited. In other words, the active yaw strategy can deal with the fluctuating nature of the wind. 

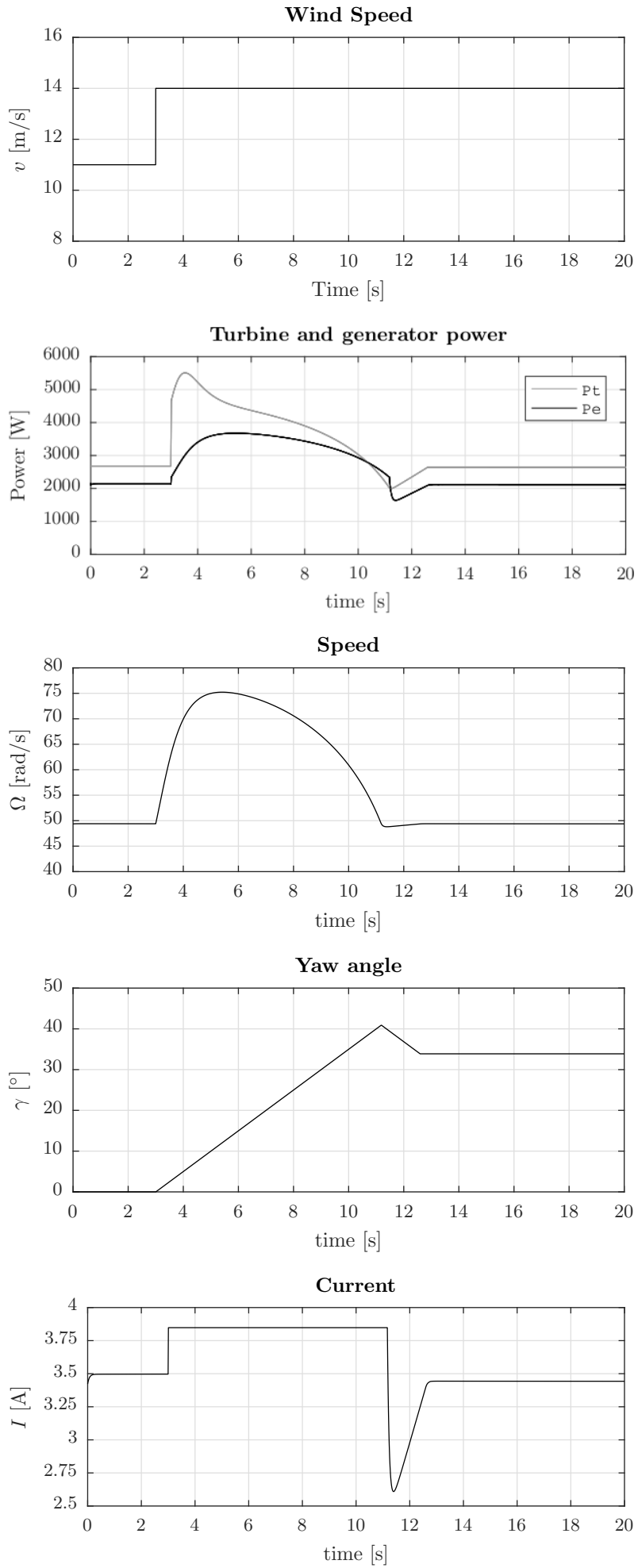

Fig. 8. Step response simulation

\section{Conclusion}

This paper proposes the use of an active yaw system to protect SMWTs in the high wind zone from overloading. A yaw controller was configured which uses on-off control with a dead zone to control the yaw angle. A supplementary rotor speed control was necessary to avoid static instability. Dynamic
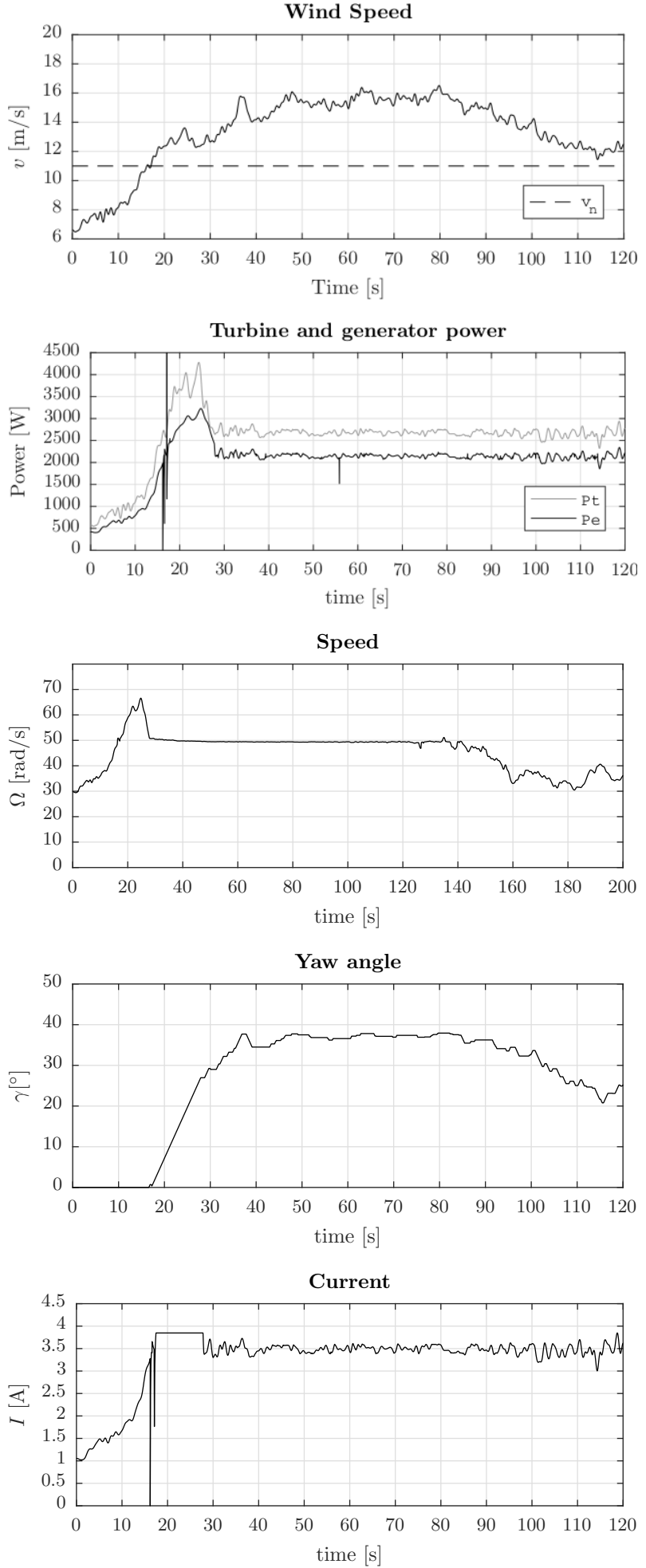

Fig. 9. Realistic simulation

simulations were performed to test the active yaw strategy for its power limiting ability. Dynamic performance was measured using the $2 \%$ settling time applied to step response simulations. A study of the settling time in function of the yaw rate rendered that yaw rates of the order $3 \%$ are required to obtain acceptable settling times. Finally, dynamic simulations using 
realistic wind profiles were performed. From these simulations it was observed that the active yaw strategy could cope with the fluctuating nature of the wind.

\section{REFERENCES}

[1] Z. Chen, J. M. Guerrero, F. Blaabjerg, "A review of the state of the art of power electronics for wind turbines," IEEE Transactions on Power Electronics, vol. 24, pp. 1859 - 1875, Aug. 2009.

[2] Y. Liu, Y. Zhao, C. Hou, G. Meng, "Research on yaw control system of $1.5 \mathrm{mw}$ double-fed wind power generators," 2016.

[3] Jiawei Chen, Jie Chen, C. Gong, "New overall power control strategy for variable-speed fixed-pitch wind turbines within the whole wind velocity range," IEEE Transactions on Industrial Electronics, vol. 60, pp. 2652-2660, 2012.

[4] J. G. Slootweg, S. W. H. de Haan, H. Polinder and W. L. Kling, "General model for representing variable speed wind turbines in power system dynamics simulations," IEEE Transactions on Power Systems, vol. 18, pp. 144-151, Feb. 2003.

[5] V. Agarwal, R. K. Aggarwal, P. Patidar and C. Patki, "A novel scheme for rapid tracking of maximum power point in wind energy generation
[5] V. Agarwal, R. K. Aggarwal, P. Patidar and C. Patki, "A novel scheme for rapid tracking of maximum power point in wind energy generation systems," IEEE Transactions on Energy Conversion, vol. 25, pp. 228236, Mar. 2010

[6] Z. Wu and H. Wang, "Research on active yaw mechanism of small wind turbines," Energy Procedia, vol. 16, pp. 53-57, 2012.

[7] F. Bu, W. Huang, Y. Hu, Y. Xu, K. Shi, and Q. Wang, "Study and implementation of a control algorithm for wind turbine yaw control system," 2009 World Non-Grid-Connected Wind Power and Energy Conference, vol. 2, no. 3, pp. 1-5, 2009.

[8] H. Choi, J. Kim, J. Cho, and Y. Nam, "Active Yaw Control of MW class Wind Turbine," International Conference on Control, Automation and Systems, pp. 1075-1078, 2010.

[9] S. Wan, L. Cheng, and X. Sheng, "Effects of yaw error on wind turbine running characteristics based on the equivalent wind speed model," Energies, vol. 8, no. 7, pp. 6286-6301, 2015.

[10] A. Mesemanolis and C. Mademlis, "Combined maximum power point and yaw control strategy for a horizontal axis wind turbine," 2014 International Conference on Electrical Machines (ICEM), pp. 17041710, 2014

[11] T. Maeda, Y. Kamada, J. Suzuki, and H. Fujioka, "Rotor Blade Sectional Performance Under Yawed Inflow Conditions," Journal of Solar Energy Engineering, vol. 130, no. 3, p. 031018, 2008. 